\title{
X-ray Photoelectron Spectroscopy with Electrical Modulation Can Be Used to Probe Electrical Properties of Liquids and Their Interfaces at Different Stages
}

\author{
Can Berk Uzundal, ${ }^{\dagger}$ Ozgur Sahin, ${ }^{\dagger}$ Pinar Aydogan Gokturk, ${ }^{\dagger, \S \odot}$ Hao Wu, ${ }^{\ddagger \odot}$ Frieder Mugele, \\ Burak Ulgut, ${ }^{\dagger}$ and Sefik Suzer* ${ }^{\dagger}+$ (이 \\ ${ }^{\dagger}$ Department of Chemistry, Bilkent University, 06800 Ankara, Turkey \\ ${ }^{\ddagger}$ Physics of Complex Fluids, University of Twente, 7522 NB, Enschede, The Netherlands
}

\section{Supporting Information}

\begin{abstract}
Operando X-ray photoelectron spectroscopy (o-XPS) has been used to record the binding energy shifts in the $\mathrm{C} 1 \mathrm{~s}$ peak of a pristine poly(ethylene glycol) (PEG) liquid drop in an electrowetting on dielectric (EWOD) geometry and after exposing it to several high-voltage breakdown processes. This was achieved by recording XPS data while the samples were subjected to $10 \mathrm{~V}$ dc and ac (square-wave modulation) actuations to extract electrical information related to the liquid and its interface with the dielectric. Through analysis of the XPS data under ac actuation, a critical frequency of $170 \mathrm{~Hz}$ is extracted for the pristine PEG, which is translated to a resistance value of $14 \mathrm{M} \Omega$ for the liquid and a

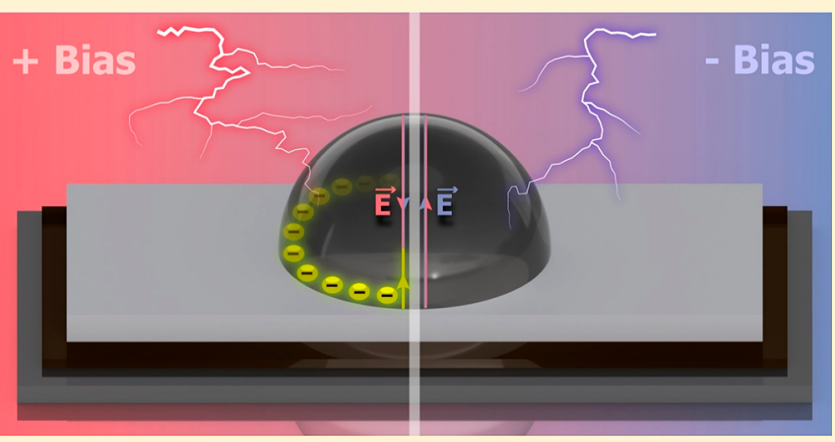
capacitance value of $60 \mathrm{pF}$ for the dielectric, by the help of simulations using an equivalent circuit model and also by XPS analyses of a mimicking device under similar conditions. The same measurements yield an increased value of $23 \mathrm{M} \Omega$ for the resistance of the liquid after the breakdown by assuming that the capacitance of the dielectric stays constant. In addition, an asymmetry in polarity dependence is observed with respect to both the onset of the breakdown voltage and also the leakage behavior of the deteriorated (PEG + dielectric) system such that deviations are more pronounced at positive voltages. Both dc and ac behaviors of the postbreakdown system can also be simulated, but only by introducing an additional element, a diode or a polarity- and magnitude-dependent voltage source (VCVS), which might be attributed to negative charge accumulation at the interface. Measurements for a liquid mixture of PEG with $8 \%$ ionic liquid yields an almost 2 orders of magnitude smaller resistance for the drop as a result of the enhanced conductivity by the ions. Coupled with modeling, XPS measurements under $\mathrm{dc}$ and ac modulations enable probing unique electrochemical properties of liquid/solid interfaces.
\end{abstract}

\section{INTRODUCTION}

X-ray photoelectron spectroscopy (XPS) is a surface-sensitive technique which has often been utilized to shed light onto chemical, physical, or structural changes following certain processes. This task is traditionally performed either ex situ, by comparing the surface composition before and after a specific process is applied, ${ }^{1-3}$ or in operando, by interrogating the surface of the sample via a designated process. ${ }^{4,5}$ Understanding electrical and electrochemical processes and/or devices under operating conditions is vital to the development of new technologies. Operando XPS with the application of an external electrical voltage bias, in either the $\mathrm{dc}$ or ac form, during data acquisition can be very useful in the investigation of such devices because of its ability to provide electrical and chemical information about the sample, which has been extensively used by our group. ${ }^{6-9}$ Extending it to the investigation of liquids has been relatively slow, mostly because of vacuum requirements, which has been successfully overcome by using multiple pumping stages and efficient electron optics. Hence, nowadays ambient pressure XPS systems are available at both synchrotron facilities and via laboratory-based instruments. ${ }^{10-20} \mathrm{~A}$ parallel investigation route has been pursued by using liquids with negligible volatility and sufficient electrical conductivity, mainly, ionic liquids and polymer electrolytes, enabling researchers to probe electrochemical properties and/or reactions with chemical specificity, rendering a wealth of electrochemical information. ${ }^{21-27}$

Our initial work in this area started with the investigation of ionic liquids as the electrolyte medium of a coplanar capacitor and using the binding energy shifts of the F 1s core level representing the anion of the ionic liquid, following polarization of one of the metal electrodes by the application of external $\mathrm{dc}$ and/or ac electrical bias. ${ }^{28}$ That investigation enabled us to follow the electrical potential developments

Received: October 9, 2019

Revised: November 28, 2019

Published: December 3, 2019 
between the metal electrodes in both laterally and temporally resolved fashions. We then extended the investigation to probing the nature of an electrochemical corrosion process between the ionic liquid and $\mathrm{Au}$ electrodes ${ }^{29}$ and finally to detecting, in situ, electrochemically generated carbene formation $^{30}$ and its $\mathrm{CS}_{2}$ adduct. ${ }^{31}$ The observation of voltage-driven asymmetric ion movement through the pores of a multilayer graphene electrode was another novel application of the operando-XPS. ${ }^{32}$ We have recently utilized Operando XPS to probe the electrowetting properties of ionic and other low-volatility liquids under both $\mathrm{dc}^{33}$ and $\mathrm{ac}^{34}$ actuation in order to derive chemically resolved electrical information on such systems, indispensably guided by electrical modeling. $^{35}$

Electrowetting is a long and heavily investigated phenomenon utilized in a wide span of scientifically and technologically important areas such as microfluidics, liquid lenses and displays, and biochemical separation and analytical sciences. $^{36-45}$ Electrowetting refers to controlling the wettability of liquids via the application of electrical forces in either the dc or ac form. ${ }^{46}$ The process has been investigated using a multitude of electrochemical, spectroscopic, and microscopic tools, ${ }^{47}$ but the use of XPS is not extensive. ${ }^{48-50}$ Our findings have reintroduced XPS as a powerful analytical arsenal for mapping the electrical voltage developments on and around liquid drops and at their interfaces to probe the effects of various electrochemical processes, in situ and in operando, similar to recent NMR studies utilized to investigate battery development, relevant to energy generation and storage. ${ }^{51,52}$

During applications of the EWOD process using aqueous solutions, two important obstacles are encountered at high applied potentials: (i) contact angle saturation and (ii) dielectric failure, both of which have been postulated to stem from the accumulation of charges at liquid/dielectric interfaces. The asymmetry with respect to the polarity of the applied potential is another phenomenon which has also been claimed to stem from the adsorption of specific ions at the liquid/ dielectric interface. ${ }^{53,54}$ It was later claimed that the same phenomena is encountered in ionic liquid interfaces and can be attributed to the enhanced electrical double-layer formation. ${ }^{55}$ However, in a recent detailed report, it was shown that the fluoropolymer-water interfaces were spontaneously and negatively charged by prolonged exposure to water and/or during the repeated application of the EWOD process. ${ }^{56}$ All of the previous works have mainly utilized surface tension or streaming potential measurements, supplemented by electrical and/or electrochemical measurements and modeling, which all provide indirect evidence for the electrical potential developments due to charge accumulation.

The present work is a continuation of our previous work, where we directly measure the electrical potential developments via recording XPS spectra of a pristine PEG drop on a Teflon-coated $\mathrm{SiO}_{2} / \mathrm{Si}$ substrate under both $\mathrm{dc}$ and ac (using square-wave pulses, SQW) low-voltage $(10 \mathrm{~V})$ actuations. Afterward, we expose the liquid drop, within the spectrometer, to higher-voltage dc actuations until it undergoes several breakdown events around $200 \mathrm{~V}$. What is different in this work from our previous papers ${ }^{33,34}$ is that we characterize the postbreakdown system again using the low-voltage actuations, which reveals yet a different asymmetric property with respect to the polarity of the actuation. This postbreakdown asymmetric behavior is different from the prebreakdown contact-angle saturation, often reported in the literature, which was also mentioned above. However, as we will discuss below, both may very well stem from the same physical/ chemical routes.

As in our previous work, our measurements at every step are guided by suitably chosen equivalent-circuit models to ensure the faithful simulation of the measured XPS data. Further validation of the XPS measurements has also been carried out on mimicking devices fabricated by using only solid-state resistors, capacitors, and, for the first time, a diode. In our previous papers, we employed either dc or ac actuations separately. The present work combines them and demonstrates, in a more comprehensive and detailed way, the synergistic use of this noninvasive spectroscopic and modeling approach, especially for uncoupling the properties of the liquid and the dielectric under different circumstances, such as during breakdown and in the presence of impurities or additives. Extending the analyses and simulation to the postbreakdown system has not been reported before and brings about additional complementary information, especially when considering the fact the low-volatility liquids used in this work, PEG, and ionic liquids are frequently employed as the liquid and/or gel-like electrolyte medium in battery systems ${ }^{57}$ or in ionic conductors such as ionic skin ${ }^{58,59}$ and neuronal scaffolds. ${ }^{60}$

The ultimate aim is to establish a new perspective for controlling and/or circumventing the unwanted. The combined methodology enables us to extract unique chemical and electrical properties of both the liquid and the dielectric under diverse conditions, derivable from no other techniques.

\section{EXPERIMENTAL SECTION}

Poly(ethylene glycol) (PEG) with $600 \mathrm{amu}$ was purchased from Sigma-Aldrich. Ionic liquid $N, N$-diethyl- $N$-methyl- $N$-(2-methoxyethyl)-ammonium tetra-fluoroborate was purchased from Io-Li-Tec. Czochralski (CZ)-grown p-type (100)-oriented silicon wafers are used as the bottom electrode. $\mathrm{A} \mathrm{SiO}_{2}$ layer with a thickness of $300 \mathrm{~nm}$ is grown on the doped silicon substrate via low-pressure chemical vapor deposition (LPCVD, Tempress) at $1100{ }^{\circ} \mathrm{C}$ for $4.5 \mathrm{~h}$. A positive-type photoresist combined with a lithography process is used to prepare a protecting layer with the exposure of the $5 \mathrm{~mm} \times 5 \mathrm{~mm}$ electrode area. $\mathrm{SiO}_{2}$ material in the exposed electrode area is etched out by a buffered oxide etch (BOE or buffered HF). The wafer substrates are cut into $3 \mathrm{~cm} \times 1.7 \mathrm{~cm}$ rectangular samples by a wafer dicing machine. The samples are cleaned with $99 \% \mathrm{HNO}_{3}$ for 2 min and rinsed with purified water afterward. A Teflon 1600 (Chemours, USA) solution is used to prepare the hydrophobic layer via a spincoating process with a spinning speed of $1500 \mathrm{rpm}$. The substrates are held at $85{ }^{\circ} \mathrm{C}$ for $1 \mathrm{~min}$, following by an annealing process at $195{ }^{\circ} \mathrm{C}$ for $1 \mathrm{~h}$ to remove all of the solvent from the film.

A Thermo Fisher $\mathrm{K} \alpha \mathrm{X}$-ray photoelectron spectrometer with monochromatized X-rays of $1486.6 \mathrm{eV}$ was used to collect all data for the XPS measurements. Spectrometer pass energies of $200 \mathrm{eV}$ for the survey spectra and $50 \mathrm{eV}$ for the high-resolution spectra of all elemental spectral regions were used. The X-ray spot size for areal and line scan measurements are set at $100 \mu \mathrm{m}$. For the time-resolved XPS, involving fast data gathering, snapshot mode with a time resolution of $1 \mathrm{~s}$ was used instead of the scanning mode. Pressure in the analyzing chamber was always kept at $<10^{-8}$ mbar. The external dc bias was applied using a Keithley 2400 source meter. For pulsed and frequency-dependent ac measurements, a Stanford Research System model DS340 function generator was used. All data fittings were carried out with Avantage software on the instrument using a mixture of $30 \%$ Gaussian and 70\% Lorentzian and employing Shirley background subtraction. As was also discussed in our previous papers and explained in detail in the Supporting Information (SI) section, we extract certain electrical properties of the investigated sample, mainly 


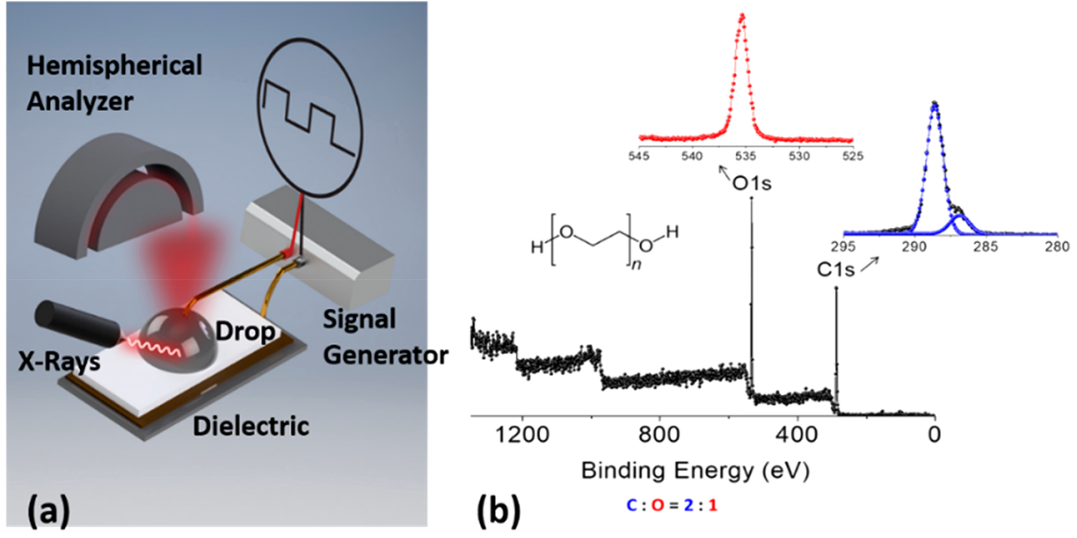

Figure 1. (a) Schematics of the experimental setup. (b) XP spectra of the pristine PEG.

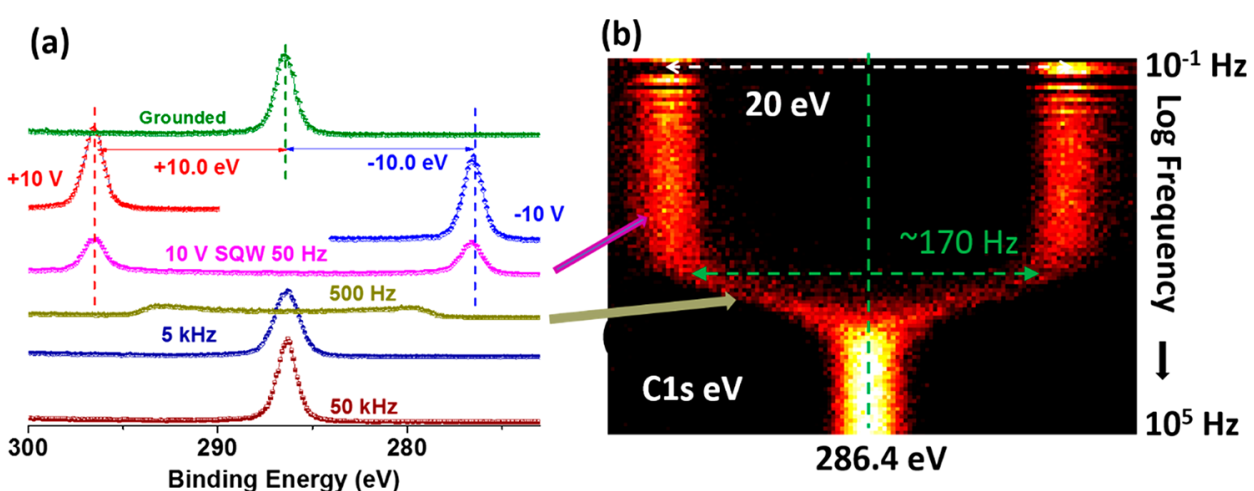

Figure 2. (a) $\mathrm{C} 1 \mathrm{~s}$ region recorded without any bias and at +10 and $-10 \mathrm{~V}$ dc bias, together with $10 \mathrm{~V} \mathrm{SQW}$ ac bias at four different frequencies. (b) Same data recorded using the snapshot mode with $1 \mathrm{~s}$ time resolution, while the drop is subjected to a logarithmic frequency sweep between $10^{-1}$ (top) and $10^{5} \mathrm{~Hz}$ (bottom). Under ac actuations, the intensity of the peak is halved because the peak is twinned. However, additional intensity changes are also encountered because of the movement of the drop, which causes differences in the electron collection efficiency of the spectrometer.

(i) resistance and (ii) developed potentials due to current passage, charge accumulation, or applied bias from the shifts in the binding energy positions of the $\mathrm{C} 1 \mathrm{~s}$ peak representing the liquid, which has a tabulated value of $286.4 \pm 0.1 \mathrm{eV}$, reflecting our detection precision of $100 \mathrm{meV}$. Accordingly, a measured $0.1 \mathrm{eV}$ shift together with a measured $10 \mathrm{pA}$ (the minimum reliable value in dc current of the power supply used) is indicative of the resistance of $10 \mathrm{G} \Omega$ (i.e., an IR drop of $0.1 \mathrm{~V}=10^{10} \Omega \times 10^{-11} \mathrm{~A}$ ), as an upper limit of the dc resistance that we can measure. A negative shift (decrease) in the binding energy corresponds to the presence of a negative voltage and vice versa.

An approximately $3 \mu \mathrm{L}$ drop of PEG (or the PEG $+8 \%$ IL mixture) was placed on top of this insulated electrode using a micropipette. A copper wire was immersed in the droplet, via which the bias was applied between the liquid and the insulated Si electrode as shown in Figure 1a. Figure 1b shows the XP spectra of the pristine PEG. For all of the measurements discussed in this work, the actuation is introduced via the wire electrode, and the bottom $\mathrm{Si}$ electrode is always grounded. All spectra presented in this article are recorded near the contact line, representative of the liquid, stemming from a depth of only $\sim 6 \mathrm{~nm}$ or less.

\section{RESULTS AND DISCUSSION}

Pristine PEG. XP spectra of the $\mathrm{C} 1 \mathrm{~s}$ region under both $\mathrm{dc}$ and ac biases are shown in Figure 2. As was presented in our previous papers, the measured binding energy shifts of the pristine PEG comply faithfully with the $10 \mathrm{~V} \mathrm{dc}$ and/or lowfrequency ac (SQW) actuations and display +10.0 or $-10.0 \mathrm{eV}$ trivial shifts in the binding energy positions because a $+10 \mathrm{~V}$ bias decreases the kinetic energy of the photoelectron by exactly $10.0 \mathrm{eV}$. Beyond a certain critical frequency, the situation differs and much smaller shifts are observed such that the liquid drop behaves like a low-pass filter (for the imposed voltage), with a critical frequency that is determined by the combined $R C$ response of the system (i.e., the liquid drop plus the dielectric layer(s)) and can be estimated using the following formula. ${ }^{61}$

$$
f_{\mathrm{c}}=\frac{\sigma_{\mathrm{l}}}{2 \pi\left(\varepsilon_{\mathrm{l}}+\varepsilon_{\mathrm{d}} \frac{R}{d}\right)}
$$

Here, $\varepsilon_{1}$ and $\varepsilon_{\mathrm{d}}$ are the permittivity of the liquid and the dielectric layer, respectively; $\sigma_{1}$ is the conductivity of the liquid; $R$ is the radius of the droplet; and $d$ is the thickness of the dielectric layer(s). Beyond the critical frequency, a fraction of the potential that is applied to the wire drops within the droplet and results in a decrease in the effective contact angle change.

For the system we use, the critical frequency is measured to be $\sim 170 \mathrm{~Hz}$, which is consistent with an estimate obtained using the geometry of the liquid and the dimensions of the Teflon and the $\mathrm{SiO}_{2}$ dielectric layers, as detailed in the SI section. In our previous work, on a similar system, this frequency was reported to be $70 \mathrm{~Hz}$, indicative of a higher capacitance for the dielectric layer. This is consistent because, therein, a much thinner ( $\sim 50 \mathrm{~nm})$ hydrophobic coating with CYTOP was utilized instead of the $1 \mu \mathrm{m}$ TEFLON coating of 


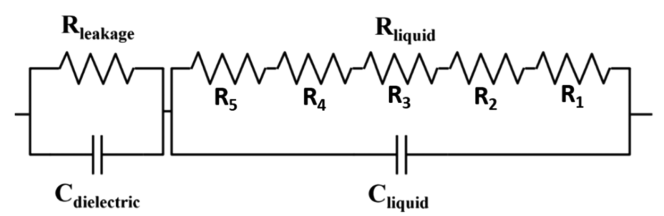

(a)

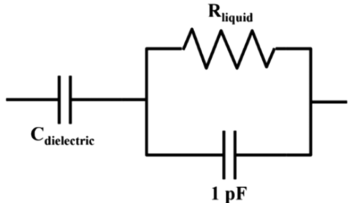

(b)

Figure 3. (a) General equivalent circuit model for the EWOD system under investigation. (b) Simplified version.

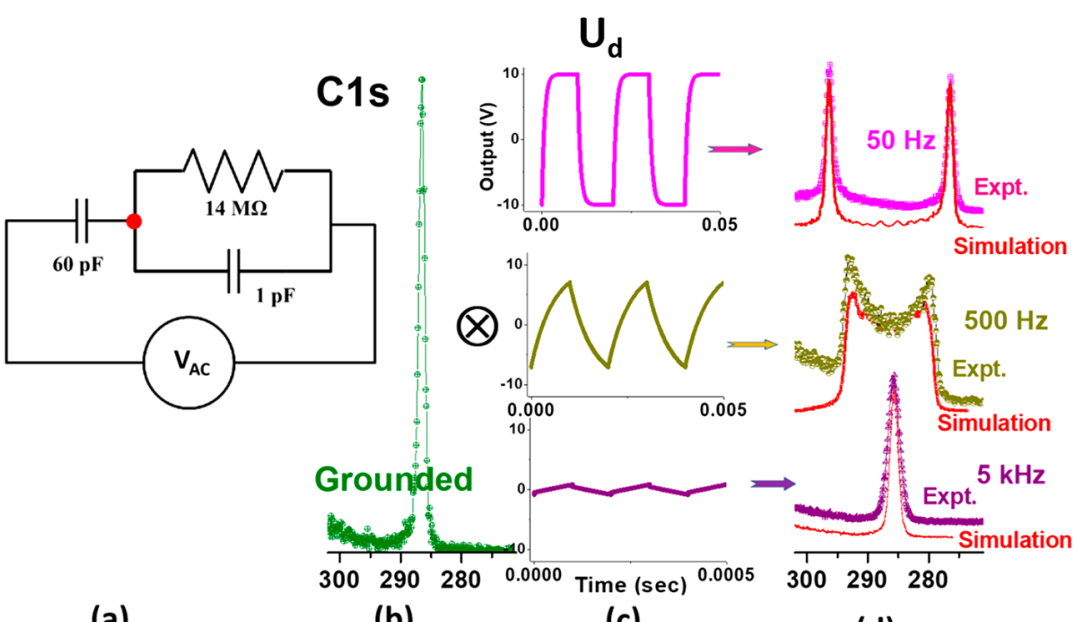

(a)

(b)

(c)

(d)

Figure 4. Ten volt SQW ac actuation and its corresponding response simulated via SPICE. (a) The simplified equivalent circuit. (b) Unbiased C 1s signal. (c) LT-Spice output waveforms $\left(\mathrm{U}_{\mathrm{d}}\right)$. (d) Spectra obtained via the convolution of the unbiased signal with the corresponding waveforms at three different frequencies, together with the experimental measurements. All data refers to those recorded/simulated at the contact line (shown as the red spot in the circuit).

this work and taking into account that the capacitance scales inversely with the thickness of the dielectric layer. In XPS measurements, the effect of the ac actuation beyond the critical frequency is manifested in the system's inability to screen and block the passage of the displacement currents, hence the decrease (increase) in the measured binding energy of the $\mathrm{C} 1 \mathrm{~s}$ peak in positive (negative) polarity (i.e., an effective IR drop).

Simulation of XP Spectra. To compare the experimentally recorded XP spectra under ac actuation, output voltage waveforms from an electrical circuit are generated with widely used simulation software, LT-Spice. ${ }^{62}$ These waveforms are used to generate the recorded XP spectra by using an in-house developed Python program, through the convolution of a lineshape function with the grounded C 1s spectrum. Following the previously published work, ${ }^{53-56,61,63,64}$ a circuit model is constructed to describe the EWOD system under investigation electrically, as shown in Figure 3a. In this circuit, the PEG droplet is represented as a capacitor $\left(C_{\text {liquid }}\right)$ in a parallel connection with a finite number (five in this case) of resistors in series $\left(R_{\text {liquid }}\right)$. In principle, one has to use an infinite number of resistors for a realistic representation of a liquid, but the finite number is chosen for practical simulation purposes. Furthermore, as was shown by numerous studies previously, the dielectric capacitance dominates the electrical response, and for a reliable dielectric, the leakage resistance can be assumed to be unmeasurable (i.e., $>10 \mathrm{G} \Omega$ in our case; see the SI section for this estimation). Therefore, the equivalent circuit can be simplified further as shown in Figure $3 b^{63,64}$

The frequency-dependent voltage response of a pristine PEG drop on Teflon can be captured by this simplified model. We assign the low time constant $R C$ to govern the response of the PEG drop and its interface with the substrate. Qualitatively analyzing the ac response of this equivalent circuit at the node on the PEG/dielectric interface, low pass filter behavior is expected for the imposed ac voltage, as was discussed in detail in ref 64 using sine-wave excitation. As a result, under lowfrequency SQW excitation no appreciable ac-current passes through the PEG drop while at high frequencies some current is expected to pass and cause an IR drop on the imposed voltage. The schematic representation of the simulation process and the results for the pristine PEG are shown in Figure 4.

Guided by the results of the simulation, together with XPS measurements on a mimicking device, fabricated by using only solid-state components, as described below and also in the SI section (Figure S7), we assign a value of $14 \mathrm{M} \Omega$ for the resistance of the liquid drop and $60 \mathrm{pF}$ for the capacitance of the dielectric layers to match the measured critical frequency of $\sim 170 \mathrm{~Hz} .{ }^{68}$ As can be seen from Figure $4 \mathrm{~d}$, this procedure and the values used faithfully reproduce the measured XP spectra recorded at 50,500, and $5 \mathrm{kHz}$, respectively. Furthermore, the determined resistance of $14 \mathrm{M} \Omega$ is quite reasonable when compared to an experimentally reported conductivity value $\left(\sim 10^{-7} \mathrm{~S} / \mathrm{m}\right)$ for the PEG-600 liquid. ${ }^{60}$ However, we must caution that only the product, $R C$, can be extracted from the experimental results.

XP Spectra under Extended dc and ac Actuations. When an external dc bias is applied to the liquid drop on the dielectric, no measurable current passes $(<10 \mathrm{pA})$, even under $\mathrm{dc}$ voltages of as large as $180 \mathrm{~V}$. As a result, both the C $1 \mathrm{~s}$ and also the $\mathrm{O}$ 1s peaks of PEG display trivial potential-dependent shifts of $180.0 \pm 0.1 \mathrm{eV}$ in their corresponding measured 


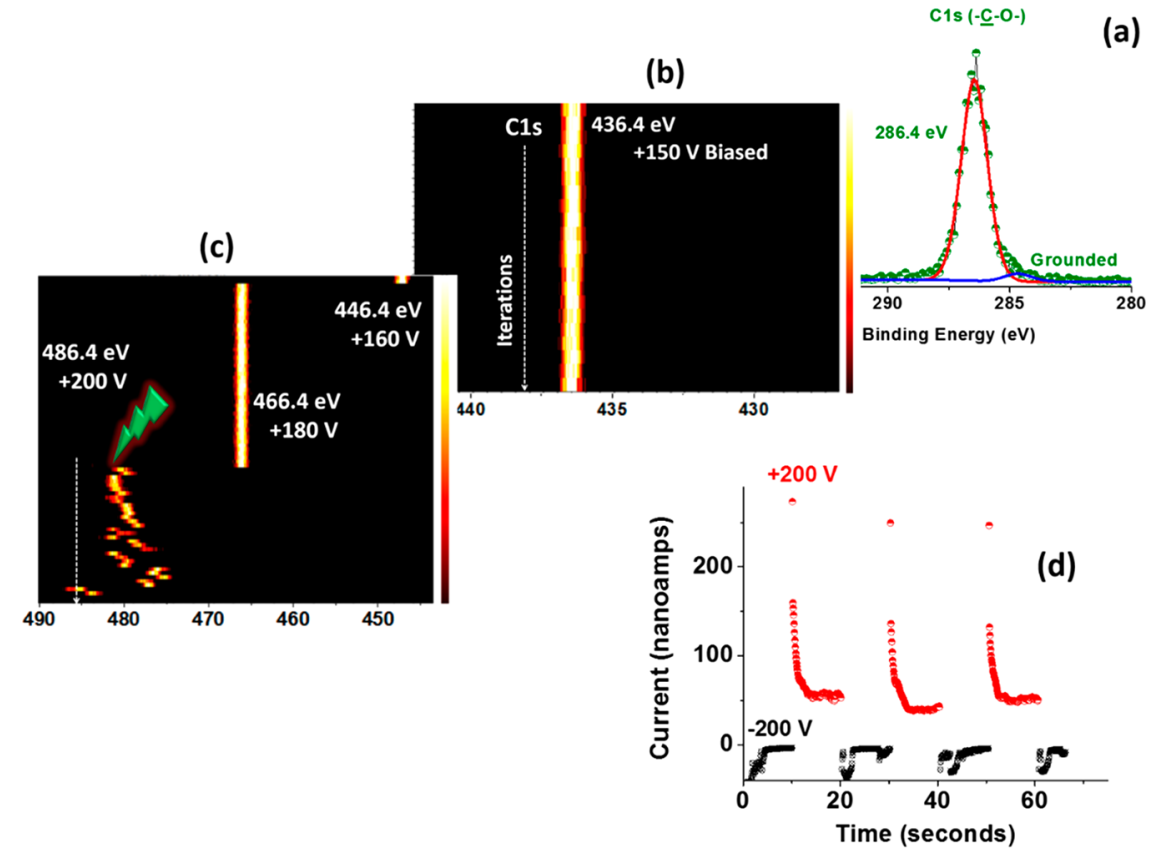

Figure 5. C 1s XP spectrum of a pristine PEG drop recorded (a) as a single region while the drop is grounded. (b) The same C 1s region is recorded iteratively under $+150 \mathrm{~V}$ as a function of time and (c) under $+160,+180$, and $+200 \mathrm{~V}$ respectively. Note that the drop undergoes several breakdown events under $+200 \mathrm{~V}$. (d) Recorded current after the breakdown events while the sample was subjected to several -200 and $+200 \mathrm{~V}$ pulses with $10 \mathrm{~s}$ intervals.

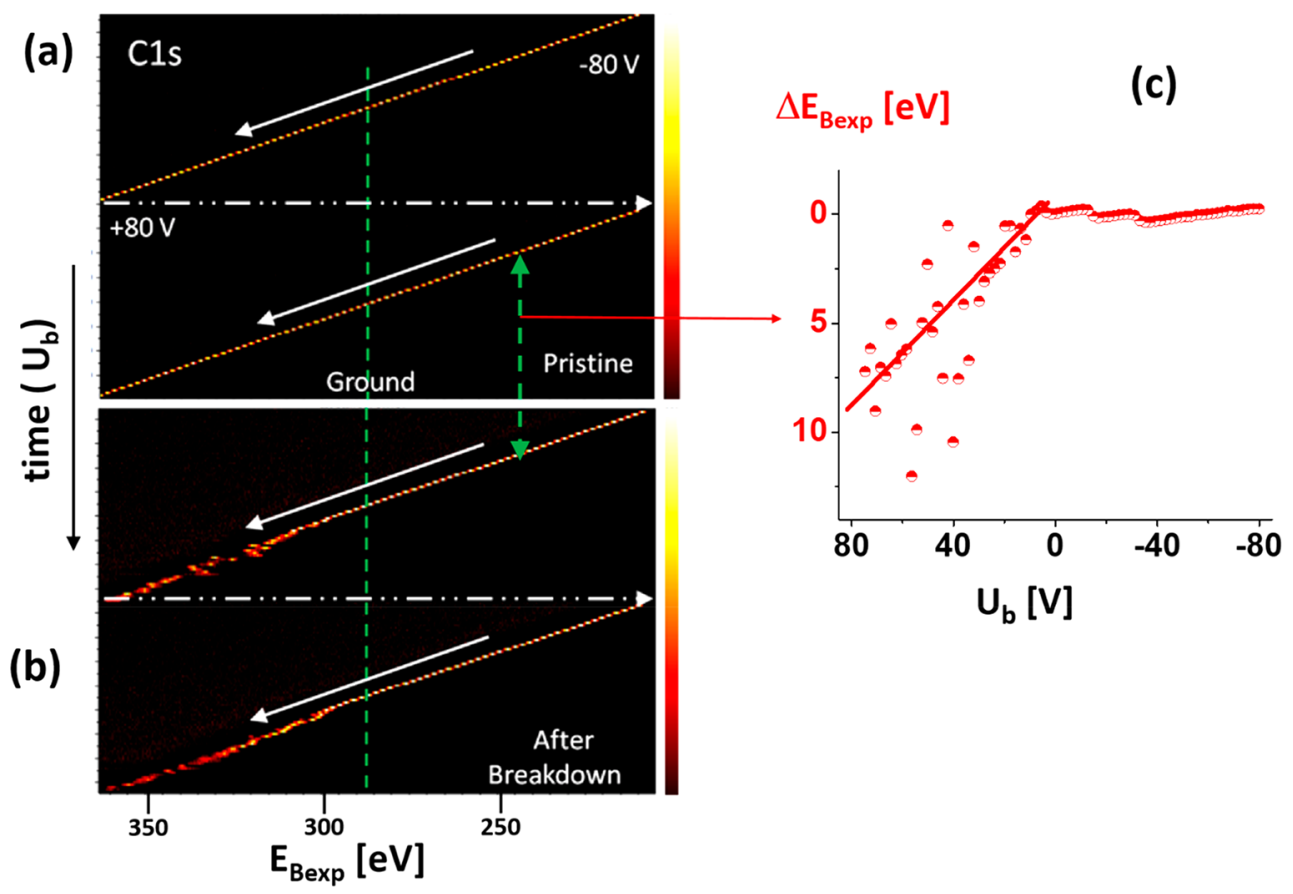

Figure 6. $\mathrm{C}$ 1s spectra recorded under slow and linear voltage sweeps from -80 to $80 \mathrm{~V}$ for (a) the pristine PEG drop and (b) the drop after breakdown. (c) The difference between the extracted binding energy values of the Pristine PEG and after breakdown $\left(\Delta E_{\mathrm{B}} \exp \right)$ as a function of the applied bias $\left(U_{\mathrm{b}}\right)$, iteratively recorded in one sweep (red half-filled dots). The red line shows the linear fit used to generate the values to be incorporated as inputs into the VCVS. (See also Figure S10 in the SI section.)

binding energies as shown explicitly in Figure 5. The estimated breakdown voltage, $180 \pm 20 \mathrm{~V}$ (see SI section for details), for this system is consistent with our measurement, which is also consistent with our previously reported measurement on a similar PEG drop on CYTOP. ${ }^{33}$

As can also be easily seen from the same figure, exposing the liquid to voltages near a breakdown threshold $<200 \mathrm{~V}$, sporadic currents ranging from 0.05 to $2 \mu \mathrm{A}$ are measurable and cause a $\mathrm{dc}$ potential drop (IR drop), which translates to lower than the expected position of the binding energy (i.e., $<486.4 \mathrm{eV}$ ), as indicated in Figure 5c. Note also that, after the first breakdown, the system displays several partial and even full recoveries, but exposure to repeated breakdown brings the drop and the dielectric couple to a state with permanently altered electrical 

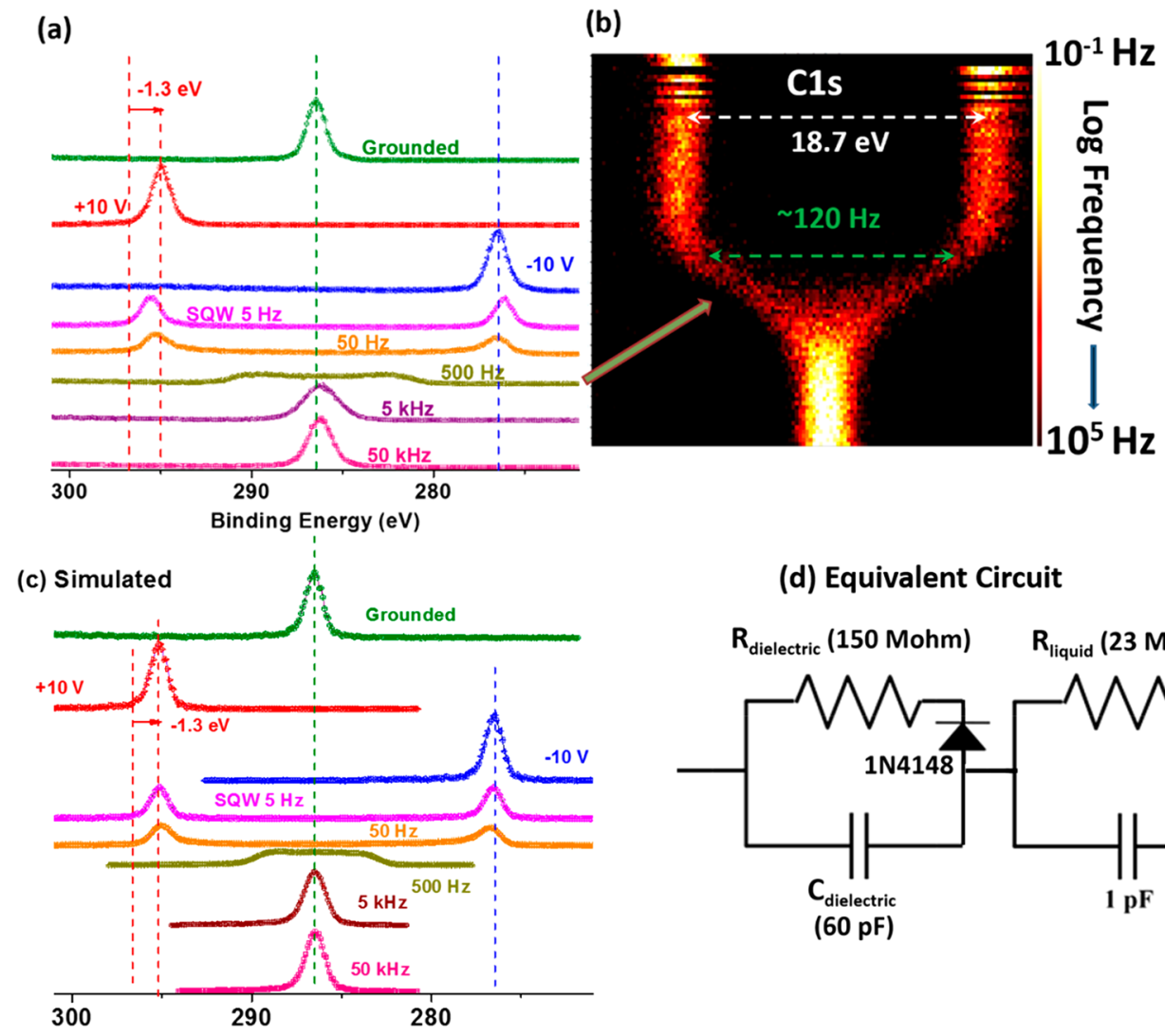

(d) Equivalent Circuit

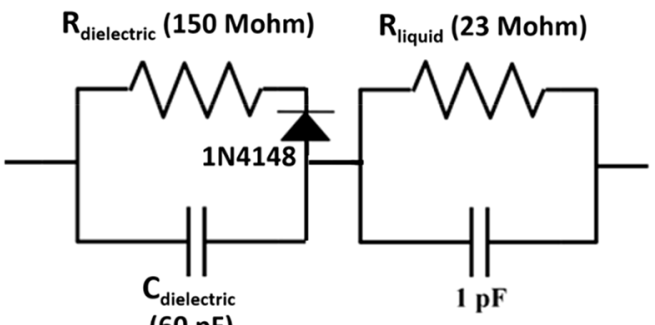

$(60 \mathrm{pF})$

Figure 7. (a) C $1 \mathrm{~s}$ region of the system after the breakdown, recorded without any bias and under +10 and $-10 \mathrm{~V}$ dc bias, together with $10 \mathrm{~V}$ SQW ac bias at four different frequencies. (b) The same data recorded using the snapshot mode with $1 \mathrm{~s}$ time resolution, while the drop is subjected to a logarithmic frequency sweep of between $10^{-1}$ (top) and $10^{5} \mathrm{~Hz}$ (bottom). (c) The same spectra as in (a), obtained from simulations using the equivalent circuit shown in (d).

properties, as can be seen from the asymmetric dc current measurements displayed in Figure $5 \mathrm{~d}$, recorded after the breakdown.

Electrical behavior of the system after breakdown is better probed by application of a repeated sawtooth excitation, slowly varying $(\sim 3 \mathrm{mHz})$ between -80 and $+80 \mathrm{~V}$ while continuously recording the $\mathrm{C}$ 1s peak $\left(E_{\mathrm{B} \exp }\right)$ as depicted in Figure 6. A careful analysis of Figure 6 reveals two important findings. Whereas only trivial voltage shifts are observable in the pristine system, the system after breakdown displays significant variations in the measured peak positions and, more importantly, only at positive voltages. The deviations are much more visible in the recorded binding difference $\left(\Delta E_{\mathrm{B} \text { exp }}\right)$ between the pristine PEG components and after its breakdown, as displayed in Figure 6c.

The asymmetric behavior of the system after breakdown is further supported through the XPS data recorded using the low voltage dc and ac actuations, which are displayed in Figure 7, better revealing the changes in the electrical properties. For example, under $+10 \mathrm{~V}$ dc bias, $\sim 56 \mathrm{nA}$ (measured) of leakage current passes through the system, causing a $-1.3 \mathrm{eV}$ shift (only a $+8.7 \mathrm{eV}$ shift is measured instead of the trivial +10.0 $\mathrm{eV}$ ) in the binding energy (IR drop of $1.3 \mathrm{~V}$ ) after the breakdown. This measurement implies that the rest of the voltage drops on the dielectric. Using this measured dc current value, XPS measurements allow us to assign $\left(1.3 \mathrm{~V} / 56 \times 10^{-9}\right.$ $\mathrm{A}=) \sim 23$ and $\sim 150 \mathrm{M} \Omega$ resistance values to the liquid and the leaky dielectric, respectively, but only at the positive polarity. We must emphasize once more that, although a small amount of current $(\sim 1 \mathrm{nA})$ is measured, no significant shifts are observed under $-10 \mathrm{~V}$ bias, which translates to a resistance value $(56 / 1 \times 155 \mathrm{M} \Omega=)$ of $\sim 9 \mathrm{G} \Omega$. The overall system after the breakdown behaves like a diode under dc actuations.

In addition to the asymmetric behavior with respect to the polarity of the bias, the critical frequency is also lowered to 120 $\mathrm{Hz}$ (see also Figure S5 in the SI section) so that application of the $10 \mathrm{~V}$ SQW actuations induces additional ac current passing through the system even at $50 \mathrm{~Hz}$. A parallel decrease in the critical frequency was also confirmed using electrochemical impedance spectroscopy (EIS) measurements on the same system (not shown). This can be attributed to an overall increase in the liquid's resistance (decrease in conductivity) because the $R C$ is also expected to increase if one assumes that the capacitance of the dielectric is not altered, as formulated in eq 1 above. An increase in the resistance is an unexpected result which will be discussed further in the article.

These two facts, the polarity-dependent leakage behavior and the lower critical frequency measured after breakdown, cannot be explained by a simple argument, such as an increase in the resistance of the liquid or the formation of a simple leaky dielectric because the latter possibility was ruled out through the XPS measurements on another mimicking device. Basically, the addition of a large leakage resistor would indeed cause less than $10.0 \mathrm{eV}$ shifts in the binding energy positions, but it works symmetrically for both the positive and negative cycles, as shown in Figure S9 of the SI section.

The aforementioned diode behavior was observed earlier using similar XPS measurements of a thick oxide $(\sim 400 \mathrm{~nm}$ 
$\mathrm{SiO}_{2}$ ) layer on $\mathrm{Si}$, an entirely solid-state system, under different surface charging conditions using a low-energy electron flood gun neutralizer. ${ }^{65}$ The behavior of the silicon oxide dielectric layer was successfully captured using again the LT-Spice program in that work, but one must keep in mind that, therein, the source of the current passing through the system was triggered solely by an external source (the flood gun). Nevertheless, we have constructed another mimicking device having a commercial Si diode (1N4148) and carried out XPS measurements to find out that the asymmetric behavior can indeed qualitatively be captured as depicted in Figure S9 in the SI section. We have also carried out simulations for the $\mathrm{C} 1 \mathrm{~s}$ spectra under $\mathrm{dc}$ and ac actuations, and the results are shown in Figure $7 c$, together with the experimental data for the system after the breakdown. The measured C 1s spectra of the system after the breakdown in Figure $7 \mathrm{a}$ and the simulated ones in Figure $7 \mathrm{c}$ are surprisingly but maybe fortuitously similar. If correct, our findings might suggest that a diode could represent either the electrical double layer at the PEG/Teflon interface or the leaky $\mathrm{SiO}_{2}-\mathrm{Si}$ underlayer, acting like an MOS capacitor. $^{66}$

An alternative argument would be to postulate the formation of an opposing negative voltage due to the accumulation of excess negative charges on the surface of the dielectric, only under positive potentials, in agreement with the findings and claims of numerous workers in this field to account for the decrease in the measured contact angle variations and/or surface tension at the liquid interface. To incorporate the development of such polarity-dependent potentials $\left(U^{*}\right)$ into our equivalent circuit model and simulate the asymmetric (diode-like) results, we introduce an additional circuit component, a voltage controlled voltage source (VCVS) as outlined in detail below and also in the SI section. Indeed, such a model was constructed by Ralston and co-workers to account for the observed deviations of the electrowetting response of aqueous ionic solutions only at positive potentials, where the actual applied voltage, $U_{\mathrm{b}}$, was modified as $\left[U_{\mathrm{b}}-U^{*}\right]$ to correct the ideal voltage at the liquid/solid interface. ${ }^{54,55}$ By using their contact angle deviations, they determined this voltage shift to $U^{*}=35 \mathrm{~V}$ at $U_{\mathrm{b}}=140 \mathrm{~V}$ to represent the energy loss during charging. More recently, Banpurkar et al. ${ }^{56}$ and Bonfante et al. ${ }^{67}$ have extended this approach to shed further light on the charging/polarization mechanisms of materials at interfaces under both $\mathrm{dc}$ and ac actuations, again using contact angle and electrical measurements.

Simulation of the Asymmetry under Both dc and ac Actuations Using a VCCS. It is possible to implement different sources using operational amplifiers (OpAmps). For instance, an example of a VCVS that multiplies the input voltage by a certain value and outputs another voltage is an OpAmp built with inverting/noninverting amplifier geometry. ${ }^{68}$ Similarly, the same noninverting amplifier can be thought of as a voltage-controlled current source (VCCS) because the current passing over the feedback path of the noninverting amplifier is directly controlled by the ratio of the input voltage with the gain resistance. The described VCCS is a common approximation to the current response of a saturated transistor at varying collector-emitter voltages. In fact, a simple power supply can be thought of as a VCVS. More complex behaviors than described herein, that capture and model fine details of various systems employing equivalent circuit models containing VCVS, can be found in the literature. For instance, the response of an array of metallic nanoparticles was modeled using a VCVS with an exponential output, ${ }^{69}$ while a mimic for second-order autonomous systems was constructed by equivalent circuits containing both VCVS and VCCS elements. ${ }^{70}$ Though the current work uses the VCVS in a simple manner to represent the diode-like behavior of the system, the arbitrary/programmable nature of the component makes it suitable for a wide range of applications. The data displayed in Figures 6 and 7 might possibly tell us that an opposing negative voltage $\left(U^{*}\right)$ develops because of negative charges trapped on Teflon as soon as the polarity becomes positive and increases approximately linearly with applied voltage. The magnitude of the opposing bias can be directly taken from the slow sweep measurements as was shown in Figure 6. The slow sweep quantifies the amount of deviation from the expected trivial shift at every applied potential and forms the output voltage of VCVS. The VCVS cuts the positive actuations with this predetermined amount such that a $10 \mathrm{~V}$ symmetric SQW (that alternates between +10 and $-10 \mathrm{~V}$ ) is reflected in the system as an asymmetric SQW (that alternates between $\left[+10-U^{*}\right]$ and $\left.-10 \mathrm{~V}\right)$. This approach allows the faithful modeling of the frequency-dependent response of the system after breakdown as shown in Figure 8, compared with the recorded XPS data. Details of the model are given in the SI section, and the results are summarized in Table 1.

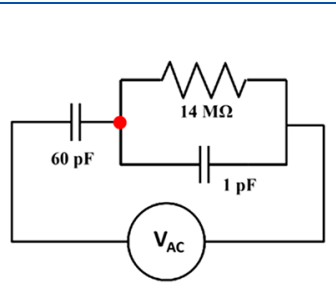

(a) Pristine PEG

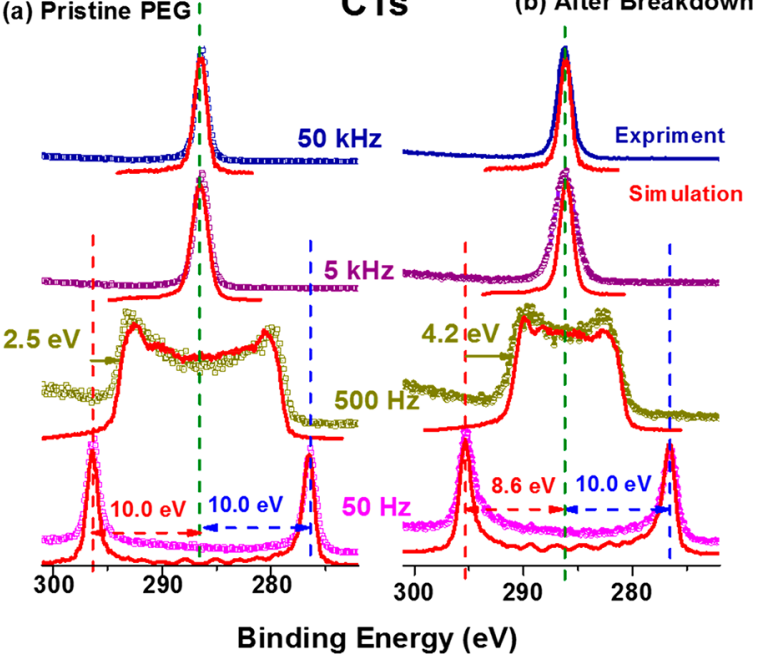

Figure 8. C 1s spectra recorded at various $10 \mathrm{~V}$ SQW ac actuations and their corresponding responses simulated via SPICE. (a) Pristine PEG. (b) After breakdown.

A physical meaning is attributable to each component in this equivalent circuit. For instance, each component in the equivalent circuit for the pristine PEG as shown in Figure 3 has a physical significance (i.e., they are related to the properties of either the dielectric or the liquid). VCVS in our equivalent circuit for the system after breakdown, consistent with literature reports demonstrating negative charge accumulation on especially the periphery of the electrolyte-Teflon interface $^{71}$ captures the electrical response of these charge 
Table 1. Extracted Data

\begin{tabular}{|c|c|c|c|c|c|c|}
\hline & \multicolumn{2}{|c|}{ liquid } & \multicolumn{2}{|l|}{ dielectric } & \multicolumn{2}{|c|}{ EWOD system critical frequency } \\
\hline & dc resistance $(R)$ & $\begin{array}{l}\text { capacitance } \\
\text { (C) }\end{array}$ & dc resistance $(R)$ & $\begin{array}{l}\text { capacitance } \\
\text { (C) }\end{array}$ & measured & $\begin{array}{c}\text { calcd } \\
(1 / 2 \pi R C)^{a}\end{array}$ \\
\hline PEG & $14 \mathrm{M} \Omega$ & $1 \mathrm{pF}$ & $>10 \mathrm{G} \Omega$ & $60 \mathrm{pF}$ & $170 \pm 20 \mathrm{~Hz}$ & $200 \mathrm{~Hz}$ \\
\hline mimicking device & $20 \mathrm{M} \Omega$ & $1 \mathrm{pF}$ & $>10 \mathrm{G} \Omega$ & $56 \mathrm{pF}$ & $120 \pm 20 \mathrm{~Hz}$ & $142 \mathrm{~Hz}$ \\
\hline PEG after breakdown & $\begin{array}{l}23 \mathrm{M} \Omega(\text { at } \\
+10 \mathrm{~V})\end{array}$ & $1 \mathrm{pF}$ & $\begin{array}{l}150 \mathrm{M} \Omega(\text { at }+10 \mathrm{~V}) \text { and } 9 \mathrm{G} \Omega(\text { at } \\
-10 \mathrm{~V})\end{array}$ & $60 \mathrm{pF}$ & $120 \pm 20 \mathrm{~Hz}$ & $142 \mathrm{~Hz}$ \\
\hline $\begin{array}{l}\underset{(1 \mathrm{~N} 4148)}{\operatorname{mimicking} \text { device }}+\text { diode } \\
\end{array}$ & $\begin{array}{l}20 \mathrm{M} \Omega(\text { at } \\
+10 \mathrm{~V})\end{array}$ & $1 \mathrm{pF}$ & $100 \mathrm{M} \Omega$ & $56 \mathrm{pF}$ & $120 \pm 20 \mathrm{~Hz}$ & $142 \mathrm{~Hz}$ \\
\hline $\mathrm{PEG}+\mathrm{IL}$ & $240 \mathrm{k} \Omega$ & $1 \mathrm{pF}$ & unmeasurable $^{b}$ & $60 \mathrm{pF}$ & $10 \pm 2 \mathrm{kHz}$ & $11 \mathrm{kHz}$ \\
\hline PEG + IL after breakdown & $\begin{array}{c}480 \mathrm{k} \Omega(\text { at } \\
+10 \mathrm{~V})\end{array}$ & $1 \mathrm{pF}$ & unmeasurable $^{b}$ & $60 \mathrm{pF}$ & $5 \pm 1 \mathrm{kHz}$ & $5.5 \mathrm{kHz}$ \\
\hline
\end{tabular}

${ }^{a}$ Frequency at which the signal drops by 3 decibels. ${ }^{68}$ In our case, this corresponds to the measured frequency at which the binding energy position drops from a $10 \mathrm{eV}$ difference to $8.5 \mathrm{eV}$. ${ }^{b}$ The lowered $\mathrm{dc}$ resistance of the liquid makes any IR drop unmeasurable because $240 \mathrm{k} \Omega \times 10 \mathrm{nA}=$ $0.024 \mathrm{eV}$ is smaller than $0.1 \mathrm{eV}$, which is our precision in binding energy measurements.

traps. In this sense, the charges trapped at the interface form a counter electric field with respect to the major field induced on the device by the electrical actuation, causing a lower-thanexpected voltage drop on the dielectric. We can use the measured opposing voltage, $U^{*}=-9.96 \mathrm{~V}$, extracted from the data in Figure 6c (and also in Figure S10a in the SI section) at $U_{\mathrm{b}}=+80 \mathrm{~V}$ to estimate the negative charge density as $\sim 0.2$ $\mathrm{mC} / \mathrm{m}^{2}$ (details are given in the SI section), in agreement with the previously reported values for similar EWOD arrangements using an aqueous medium as the liquid. ${ }^{54,55}$ The difference, however, lies in the fact that PEG, being prominently a dipolar liquid, cannot provide the ions for the charge trapping. Therefore, the dielectric surface (Teflon) might be the source of such negative charges trapped.

PEG + $8 \%$ Ionic Liquid. Because liquid poly(ethylene glycol) has a predominantly dipolar structure, the addition of ionic moieties such as organic and/or inorganic ions is expected to impart large changes to its electrical properties, as was reported in our previous work. ${ }^{33}$ A mixture of PEG, containing $\sim 8 \%$ (by weight) ionic liquid consisting of a tertiary amine as the cation and tetrafloroborate $\left(\mathrm{BF}_{4}{ }^{-}\right)$as the anion, has also been subjected to similar XP measurements. The results are displayed in Figure 9, and the relevant extracted data are given in Table 1. As expected, the ionic content of the liquid imparts an almost 2 orders of magnitude jump in conductivity but has little to no effect on the electrical properties of the dielectric layer(s).

These results differ from our earlier measurements on the same liquid PEG containing an all-inorganic $\mathrm{MgCl}_{2}$ salt, where only an $\sim 50 \%$ increase was observed in the conductivity, which also did not exhibit any measurable dependence on the salt's concentration. ${ }^{34}$ Those findings were attributed to the limited solubility of $\mathrm{MgCl}_{2}$ within PEG, in accordance with the previously published data reported by Di Noto et al. ${ }^{72,73}$ It is worth mentioning that the large increase in the conductivity (decrease in the resistance to levels of $\sim 100 \mathrm{k} \Omega$ ) of the liquid makes it impossible to determine the voltage offsets, if any, due to the accumulation of charges by XPS under dc actuations. However, the critical frequency changes via ac actuations nevertheless yield useful electrical information.

\section{CONCLUSIONS}

We have recorded XP spectra of a nonvolatile liquid drop configured in an electrowetting on dielectric geometry and under both low voltage $\mathrm{dc}$ and ac actuations before and after it was forced to undergo several dielectric breakdown events at
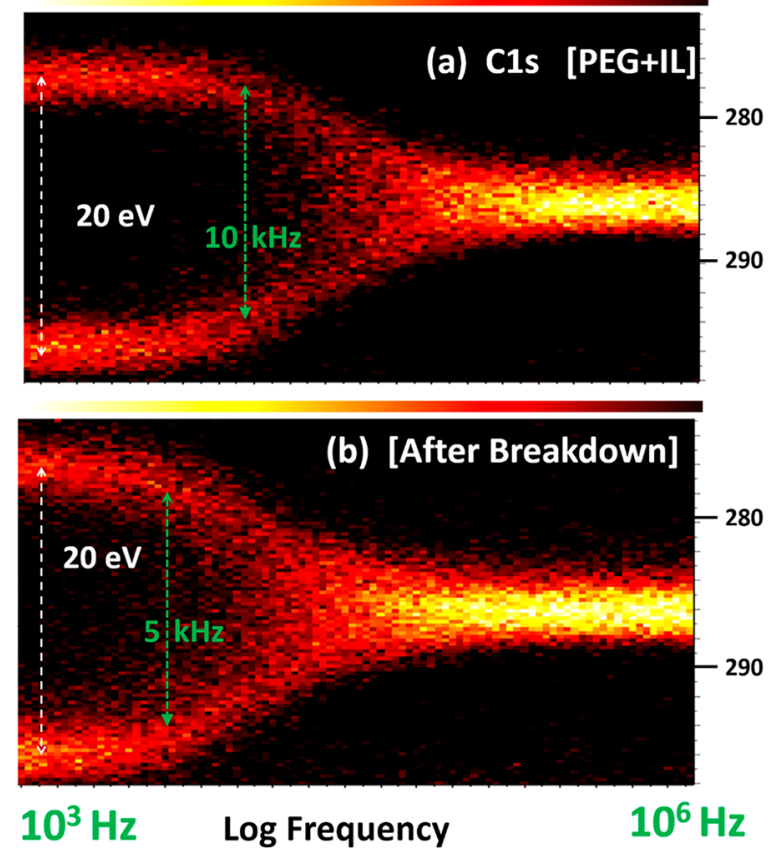

Figure 9. C 1s spectra recorded at various $10 \mathrm{~V}$ SQW ac actuations and their corresponding responses simulated via SPICE. (a) Pristine PEG. (b) After breakdown.

larger voltages. Using the measured shifts in the binding energy position of the $\mathrm{C} 1 \mathrm{~s}$ peak representing the liquid, we extract local potentials in a totally noninvasive way, which are translated to electrical properties of the liquid and its interface with the dielectric substrate, by the help of equivalent circuit analyses and simulations. The simulation results are further validated with the help of XPS analyses of mimicking devices constructed using all-solid-state elements, such as resistors, capacitors, and a diode. This unique synergistic combination of an XPS analyses + simulations + mimicking approach yields several unexpected features, such as the abilities (i) to separate the electrical properties of the liquid drop from those of the dielectric and under diverse conditions, (ii) to determine a decrease in the conductivity of the liquid after its breakdown, which is contrary to a common understanding since strong electrical disturbances are usually expected to cause fragmentation and/or produce additional ionic moieties and hence increase the conductivity, and (iii) to expose the 
asymmetric behavior of the postbreakdown system with respect to the polarity of the applied potential.

To account for and simulate this new asymmetric postbreakdown behavior using an equivalent-circuit model, we were forced to incorporate, in an ad-hoc fashion, a diode possibly representing the liquid/dielectric interface, or the $\mathrm{SiO}_{2} / \mathrm{Si}$ dielectric underlayer, or a VCVS representing polaritydependent opposing voltage developments at the liquid/ dielectric interface. Further experimental and simulation work is definitely needed to elucidate the physical-chemical nature of the observed relations and their implications for the electrified liquid/solid interfaces.

In any case, our methodology introduces a new perspective for the investigation of liquid/solid interfaces, which is very easy to implement. Therefore, we expect it to be adopted by many others in the field. We also advocate that our methodology will impact several electrochemical issues that are relevant especially for energy harvesting and storage.

\section{ASSOCIATED CONTENT}

\section{(5) Supporting Information}

The Supporting Information is available free of charge at https://pubs.acs.org/doi/10.1021/acs.langmuir.9b03134.

Details of XPS data gathering, additional data, estimation of electrical parameters of the various materials used, various equivalent circuit models, and mimicking devices (PDF)

\section{AUTHOR INFORMATION}

\section{Corresponding Author}

*E-mail: suzer@fen.bilkent.edu.tr.

\section{ORCID $\odot$}

Pinar Aydogan Gokturk: 0000-0001-8269-8884

Hao Wu: 0000-0002-8197-3335

Burak Ulgut: 0000-0002-4402-0033

Sefik Suzer: 0000-0002-5866-2600

\section{Present Address}

${ }^{\S}$ Advanced Light Source, Lawrence Berkeley National Laboratory, Berkeley, California 94720, United States.

\section{Notes}

The authors declare no competing financial interest.

\section{ACKNOWLEDGMENTS}

This work is partially supported by the Scientific and Technological Research Council of Turkey (TUBITAK) through the funds from research grant no. 118Z902.

\section{REFERENCES}

(1) Södergren, S.; Siegbahn, H.; Rensmo, H.; Lindström, H.; Hagfeldt, A.; Lindquist, S.-E. Lithium Intercalation in Nanoporous Anatase TiO2 Studied with XPS. J. Phys. Chem. B 1997, 101, 30873090.

(2) Zhou, W.; Baunach, T.; Ivanova, V.; Kolb, D. M. Structure and Electrochemistry of 4,4'-Dithiodipyridine Self-Assembled Monolayers in Comparison with 4-Mercaptopyridine Self-Assembled Monolayers on $\mathrm{Au}(111)$. Langmuir 2004, 20, 4590-4595.

(3) Pearse, A. J.; Gillette, E.; Bok Lee, S. B.; Rubloff, G. W. The reaction current distribution in battery electrode materials revealed by XPS-based state-of-charge mapping. Phys. Chem. Chem. Phys. 2016, 18, 19093-19102.
(4) Tonti, D.; Pettekofer, C.; Jaegermann, W. In-Situ Photoelectron Spectroscopy Study of a $\mathrm{TiS}_{2}$ Thin Film Cathode in an Operating $\mathrm{Na}$ Intercalation Electrochemical Cell. Ionics 2000, 6, 196-202.

(5) Thißen, A.; Ensling, D.; Liberatore, M.; Wu, Q.-H.; Madrigal, F. F.; Bhuvaneswari, M.; Hunger, R.; Jaegermann, W. Experimental Routes to in situ Characterization of the Electronic Structure and Chemical Composition of Cathode Materials for Lithium Ion Batteries During Lithium Intercalation and Deintercalation Using Photoelectron Spectroscopy and Related Techniques. Ionics 2009, 15, 393-403.

(6) Yilmaz, E.; Sezen, H.; Suzer, S. Probing the charge build-up and dissipation on thin PMMA film surfaces at the molecular level by XPS. Angew. Chem., Int. Ed. 2012, 51, 5488-5492.

(7) Sezen, H.; Suzer, S. XPS for Chemical- and Charge-Sensitive Analyses. Thin Solid Films 2013, 534, 1-11.

(8) Copuroglu, M.; Aydogan, P.; Polat, E. O.; Kocabas, C.; Süzer, S. Gate-Tunable Photoemission from Graphene Transistors. Nano Lett. 2014, 14, 2837-2842.

(9) Aydogan, P.; Balci, O.; Suzer, S. Monitoring the operation of a graphene transistor in an integrated circuit by XPS. Org. Electron. 2016, 37, 178-182.

(10) Siegbahn, H. Electron Spectroscopy for Chemical Analysis of Liquids and Solutions. J. Phys. Chem. 1985, 89, 897-909.

(11) Moberg, R.; Bökman, F.; Bohman, O.; Siegbahn, H. ESCA Studies of Phase-Transfer Catalysts in Solution: Ion Pairing and Surface Activity. J. Am. Chem. Soc. 1991, 113, 3663-3667.

(12) Bökman, F.; Bohman, O.; Siegbahn, H. ESCA Studies of Phase Transfer Catalysts in Solution. 2. Surface Ion Pairing and Salting-Out Effects. J. Phys. Chem. 1992, 96, 2278-2283.

(13) Winter, B.; Faubel, M. Photoemission from Liquid Aqueous Solutions. Chem. Rev. 2006, 106, 1176-1211.

(14) Ghosal, S.; Hemminger, J. C.; Bluhm, H.; Mun, B. S.; Hebenstreit, E. L.; Ketteler, G.; Ogletree, D. F.; Requejo, F. G.; Salmeron, M. Electron Spectroscopy of Aqueous Solution Interfaces Reveals Surface Enhancement of Halides. Science 2005, 307, 563566.

(15) Bluhm, H.; Andersson, K.; Araki, T.; Benzerara, K.; Brown, G. E.; Dynes, J. J.; Ghosal, S.; Gilles, M. K.; Hansen, H.-C.; Hemminger, J. Soft X-ray Microscopy and Spectroscopy at the Molecular Environmental Science Beamline at the Advanced Light Source. J. Electron Spectrosc. Relat. Phenom. 2006, 150, 86-104.

(16) Zhang, C.; Grass, M. E.; Yu, Y.; Gaskell, K. J.; DeCaluwe, S. C.; Chang, R.; Jackson, G. S.; Hussain, Z.; Bluhm, H.; Eichhorn, B. W. Multielement Activity Mapping and Potential Mapping in Solid Oxide Electrochemical Cells Through the use of Operando XPS. ACS Catal. 2012, 2, 2297-2304.

(17) Brown, M. A.; Redondo, A. B.; Sterrer, M.; Winter, B.; Pacchioni, G.; Abbas, Z.; van Bokhoven, J. A. Measure of Surface Potential at the Aqueous-Oxide Nanoparticle Interface by XPS from a Liquid Microjet. Nano Lett. 2013, 13, 5403-5407.

(18) Lichterman, M. F.; Hu, S.; Richter, M. H.; Crumlin, E. J.; Axnanda, S.; Favaro, M.; Drisdell, W.; Hussain, Z.; Mayer, T.; Brunschwig, B. S. Direct Observation of the Energetics at a Semiconductor/Liquid Junction by Operando X-ray Photoelectron Spectroscopy. Energy Environ. Sci. 2015, 8, 2409-2416.

(19) Brown, M. A.; Abbas, Z.; Kleibert, A.; Green, R. G.; Goel, A.; May, S.; Squires, T. M. Determination of Surface Potential and Electrical Double-Layer Structure at the Aqueous ElectrolyteNanoparticle Interface. Phys. Rev. X 2016, 6, 011007.

(20) Broderick, A.; Rocha, M. A.; Khalifa, Y.; Shiflett, M. B.; Newberg, J. T. Mass Transfer Thermodynamics through a GasLiquid Interface. J. Phys. Chem. B 2019, 123, 2576-2584.

(21) Lovelock, K. R.; Villar-Garcia, I. J.; Maier, F.; Steinrück, H.-P.; Licence, P. Photoelectron Spectroscopy of Ionic Liquid-Based Interfaces. Chem. Rev. 2010, 110, 5158-5190.

(22) Qiu, F.; Taylor, A. W.; Men, S.; Villar-Garcia, I. J.; Licence, P. An Ultra High Vacuum-Spectroelectrochemical Study of the Dissolution of Copper in the Ionic Liquid (N-methylacetate)-4- 
picolinium Bis (Trifluoromethylsulfonyl) Imide. Phys. Chem. Chem. Phys. 2010, 12, 1982-1990.

(23) Foelske-Schmitz, A.; Weingarth, D.; Kötz, R. Quasi in situ XPS Study of Electrochemical Oxidation and Reduction of Highly Oriented Pyrolytic Graphite in [1-ethyl-3-methylimidazolium][BF 4] Electrolytes. Electrochim. Acta 2011, 56, 10321-10331.

(24) Wibowo, R.; Aldous, L.; Jacobs, R. M.; Manan, N. S.; Compton, R. G. Monitoring Potassium Metal Electrodeposition From an Ionic Liquid Using in situ Electrochemical-X-ray Photoelectron Spectroscopy. Chem. Phys. Lett. 2011, 509, 72-76.

(25) Weingarth, D.; Foelske-Schmitz, A.; Wokaun, A.; Kötz, R. In Situ Electrochemical XPS Study of the Pt/[EMIM][BF 4] System. Electrochem. Commun. 2011, 13, 619-622.

(26) Fujimoto, T.; Awaga, K. Electric-Double-Layer Field-Effect Transistors with Ionic Liquids. Phys. Chem. Chem. Phys. 2013, 15, 8983-9006.

(27) Matsuda, T.; Taccardi, N.; Schwegler, J.; Wasserscheid, P.; Steinrück, H. P.; Maier, F. Vacuum Surface Science Meets Heterogeneous Catalysis: Dehydrogenation of a Liquid Organic Hydrogen Carrier in the Liquid State. ChemPhysChem 2015, 16, $1873-1879$.

(28) Olschewski, M.; Gustus, R.; Höfft, O.; Lahiri, A.; Endres, F. Monochromatic X-ray Photoelectron Spectroscopy Study of Three Different Ionic Liquids in Interaction with Lithium-Decorated Copper Surfaces. J. Phys. Chem. C 2017, 121, 2675-2682.

(29) Camci, M.; Aydogan, P.; Ulgut, B.; Kocabas, C.; Suzer, S. XPS Enables Visualization of Electrode Potential Screening in an Ionic Liquid Medium with Temporal-and Lateral-Resolution. Phys. Chem. Chem. Phys. 2016, 18, 28434-28440.

(30) Camci, M. T.; Ulgut, B.; Kocabas, C.; Suzer, S. In-Situ XPS Monitoring and Characterization of Electrochemically Prepared $\mathrm{Au}$ Nanoparticles in an Ionic Liquid. ACS Omeg. 2017, 2, 478-486.

(31) Aydogan Gokturk, P.; Salzner, U.; Nyulászi, L.; Ulgut, B.; Kocabas, C.; Suzer, S. XPS-Evidence for in-situ ElectrochemicallyGenerated Carbene Formation. Electrochim. Acta 2017, 234, 37-42.

(32) Aydogan Gokturk, P.; Donmez, S.; Ulgut, B.; Türkmen, Y.; Suzer, S. Optical and XPS Evidence for the Electrochemical Generation of an N-heterocyclic Carbene and its CS 2 Adduct from the Ionic Liquid [Bmim] [PF 6]. New J. Chem. 2017, 41, 1029910304.

(33) Camci, M. T.; Ulgut, B.; Kocabas, C.; Suzer, S. In-Situ XPS reveals Voltage Driven Assymetric Ion Movement of an Ionic Liquid through the pores of a Multilayer Graphene. J. Phys. Chem. C 2018, 122, 11883-11889.

(34) Aydogan Gokturk, P.; Ulgut, B.; Suzer, S. dc Electrowetting of a nonaqueous liquid revisited by XPS. Langmuir 2018, 34, 7301-7308.

(35) Aydogan Gokturk, P.; Ulgut, B.; Suzer, S. AC Electrowetting Modulation of Low Volatile Liquids Probed by XPS: Dipolar vs. Ionic Screening. Langmuir 2019, 35, 3319-33326.

(36) Uzundal, C. B.; Aydogan Gokturk, P.; Suzer, S.; Ulgut, B. Coarse-Grained Electrostatic Model Including Ion-Pairing Equilibrium That Explains DC and AC X-ray Photoelectron Spectroscopy Measurements on Ionic Liquids. J. Phys. Chem. C 2019, 123, 1319213200.

(37) Pollack, M. G.; Fair, R. B.; Shenderov, A. D. Electrowettingbased actuation of liquid droplets for microfluidic applications. Appl. Phys. Lett. 2000, 77, 1725-1726.

(38) Berge, B.; Peseux, J. Variable focal lens controlled by an external voltage: An application of electrowetting. Eur. Phys. J. E: Soft Matter Biol. Phys. 2000, 3, 159-163.

(39) Kuiper, S.; Hendriks, B. H. W. Variable-focus liquid lens for miniature cameras. Appl. Phys. Lett. 2004, 85, 1128-1130.

(40) Srinivasan, V.; Pamula, V. K.; Fair, R. B. An integrated digital microfluidic lab-on-a-chip for clinical diagnostics on human physiological fluids. Lab Chip 2004, 4, 310-315.

(41) Ren, H.; Fair, R. B.; Pollack, M. G. Automated on-chip droplet dispensing with volume control by electro-wetting actuation and capacitance metering. Sens. Actuators, B 2004, 98, 319-327.
(42) Chatterjee, D.; Hetayothin, B.; Wheeler, A. R.; King, D. J.; Garrell, R. L. Droplet-based microfluidics with nonaqueous solvents and solutions. Lab Chip 2006, 6, 199-206.

(43) Gong, J.; Kim, C.-J. C. All-electronic droplet generation on-chip with real-time feedback control for ewod digitial microfluidics. Lab Chip 2008, 8, 898-906.

(44) Hong, J.; Kim, Y. K.; Won, D.-J.; Kim, J.; Lee, S. J. Threedimensional digital microfluidic manipulation of droplets in oil medium. Sci. Rep. 2015, 5, 10685.

(45) Sadeghi, S.; Ding, H.; Shah, G. J.; Chen, S.; Keng, P. Y.; Kim, C.-J. C.; van Dam, R. M. On Chip Droplet Characterization: A Practical, High-Sensitivity Measurement of Droplet Impedance in Digital Microfluidics. Anal. Chem. 2012, 84, 1915-1923.

(46) Mugele, F.; Baret, J.-C. Electrowetting: from basics to applications. J. Phys.: Condens. Matter 2005, 17, R705-R774.

(47) Mugele, F.; Heikenfeld, J. Electrowetting: Fundamental Principles and Practical Applications; Wiley-VCH: Weinheim, Germany, 2019.

(48) Zeng, X.; Zhang, K.; Guowei, T.; Zeng, Z.; Fan, S. K.; Zhou, J. Recoverable electrowetting-on-dielectric device in chemiluminescence enzymatic detector. Jpn. J. Appl. Phys. 2014, 53, 060304.

(49) Zhang, K.; Chao, L.; Zhou, J. Biocompatible/Biodegradable Electrowetting on Dielectric Microfluidic Chips with Fluorinated CTA/PLGA. Materials 2018, 11, 1332.

(50) Panhwar, G. M.; Mysyk, R.; Rojo, T.; Shaikhutdinov, S.; Bondarchuk, O. Electrowetting of Ionic Liquid on Graphite: Probing via in Situ Electrochemical X-ray Photoelectron Spectroscopy. Langmuir 2018, 34, 14528-14536.

(51) Grey, C. P.; Tarascon, J. M. Sustainability and in situ monitoring in battery development. Nat. Mater. 2017, 16, 45-56.

(52) Hope, M. A.; Griffith, K. J.; Cui, B.; Gao, F.; Dutton, S. E.; Parkin, S. S. P.; Grey, C. P. The Role of Ionic Liquid Breakdown in the Electrochemical Metallization of $\mathrm{VO}_{2}$ : An NMR Study of Gating Mechanisms and $\mathrm{VO}_{2}$ Reduction. J. Am. Chem. Soc. 2018, 140, 16685-16696.

(53) Verheijen, J. J.; Prins, M. W. J. Reversible Electrowetting and Trapping of Charge: Model and Experiment. Langmuir 1999, 15, 6616-6620.

(54) Quinn, A.; Sedev, R.; Ralston, J. Influence of the Electrical Double layer in Electrowetting. J. Phys. Chem. B 2003, 107, 11631169.

(55) Millefiorini, S.; Tkaczyk, A. H.; Sedev, R.; Efthimiadis, J.; Ralston, J. Electrowetting of Ionic Liquids. J. Am. Chem. Soc. 2006, 128, 3098-3101.

(56) Banpurkar, A. G.; Sawane, Y.; Wadhai, S. M.; Murade, C. U.; Siretanu, I.; van den Ende, D.; Mugele, F. Spontaneous electrification of fluoropolymer-water interfaces probed by electrowetting. Faraday Discuss. 2017, 199, 29-47.

(57) Kwon, Y. H.; Minnici, K.; Huie, M. M.; Takeuchi, K. J.; Takeuchi, E. S.; Marschilok, A. C.; Reichmanis, E. Electron/Ion Transport Enhancer in High Capacity Li-Ion Battery Anodes. Chem. Mater. 2016, 28, 6689-6697.

(58) Keplinger, C.; Sun, J.-Y.; Foo, C. C.; Rothemund, P.; Whitesides, G. M.; Suo, Z. Strchable, Transperent, Ionic Conductors. Science 2013, 341, 984-987.

(59) Sun, J.-Y.; Keplinger, C.; Whitesides, G. M.; Suo, Z. Ionic Skin. Adv. Mater. 2014, 26, 7608-7614.

(60) Sikkema, W. K. A.; Metzger, A. B.; Wang, T.; Tour, J. M. Physical and electrical characterization of TexasPEG: An electrically conductive neuronal scaffold. Surgical Neurology International 2017, 8, 84.

(61) Hong, J. S.; Ko, S. H.; Kang, K. H.; Kang, I. S. A numerical investigation on AC electrowetting of a droplet. Microfluid. Nanofluid. 2008, 5, 263-271.

(62) https://www.analog.com/en/design-center/design-tools-andcalculators/LTspice-s.

(63) Hu, X.; Mibus, M.; Knospe, C. R.; Zangari, G.; Reed, M. L. Impedance spectroscopy and electrical modeling of electrowetting on dielectric devices. J. Micromech. Microeng. 2015, 25, 045020. 
(64) Kumar, A.; Pluntke, M.; Cross, B. J. F.; Cross, B. J. F.; Baret, J.C.; Mugele, F. G. Charged droplet generation and finite conductivity effects in AC Electrowetting. MRS Fall Meeting 2005; Faculty of Science and Technology, Physics of Complex Fluids, University of Twente.

(65) Tasci, T. O.; Atalar, E.; Demirok, U. K.; Suzer, S. Electrical circuit modelling of surface structures for X-Ray photoelectron spectroscopic measurements. Surf. Sci. 2008, 602, 365-368.

(66) Rangan, S.; Kalyanikar, M.; Duan, J.; Liu, G.; Bartynski, R. A.; Andrei, E. Y.; Feldman, L.; Garfunkel, E. Nanoscale Internal Fields in a Biased Graphene-Insulator- Semiconductor Structure. J. Phys. Chem. Lett. 2016, 7, 3434-3439.

(67) Bonfante, G.; Roux-Marchard, T.; Audry-Deschamps, M.-C.; Renaud, L.; Kleimann, P.; Brioude, A.; Mallard, M. Polarization mechanisms of dielectric materials at a binary liquid interface:impacts on electrowetting actuation. Phys. Chem. Chem. Phys. 2017, 19, 30139-30146.

(68) Malvino, A. P. Electronic Principles, 5th ed.; Macmillan/ McGraw-Hill: New York, 1993.

(69) Song, K.; Mazumder, P. An Equivalent Circuit Modeling of an Equispaced Metallic Nanoparticles (MNPs) Plasmon Wire. IEEE Trans. Nanotechnol. 2009, 8 (3), 412-418.

(70) Pospísill, J.; Kolka, Z.; Hanus, S.; Petržela, J.; Brzobohatý, J. Optimized second-order dynamical systems and their RLC circuit models with PWL controlled sources. Radioengineering 2003, 13 (3), 13-17.

(71) Kudina, O.; Eral, B.; Mugele, F. e-MALDI: An ElectrowettingEnhanced Drop Drying Method for MALDI Mass Spectrometry. Anal. Chem. 2016, 88 (9), 4669-4675.

(72) Di Noto, V.; Longo, D.; Münchow, V. Ion-Oligomer Interactions in Poly(ethylene glycol $) 400 /(\mathrm{LiCl}) x$ Electrolyte Complexes. J. Phys. Chem. B 1999, 103, 2636-2646.

(73) Di Noto, V.; Vittadello, M. Mechanism of ionic conductivity in poly(ethylene glycol 400$) /(\mathrm{MgCl} 2) \mathrm{x}$ polymer electrolytes: studies based on electrical spectroscopy. Solid State Ionics 2002, 147, 309316. 